\title{
Patrones de especialización de la investigación cubana en salud
}

\author{
Specialization Patterns in the Cuban national health research
}

\author{
Dr. C. Ricardo Arencibia J orge,' Dr. C. Rosa Lidia Vega Almeida," Dr. C. Zaida \\ Chinchilla Rodríguez," "Dr. C. Elena Corera Álvarez,"' Dr. C. Félix de Moya \\ Anegón "II \\ ' Centro Nacional de Investigaciones Científicas. La Habana, Cuba. \\ "Centro de Histoterapia Placentaria. La Habana, Cuba. \\ "I Grupo SCImago, Instituto de Políticas y Bienes Públicos, IPP-CSIC. Madrid, España.
}

\section{RESUMEN}

Introducción: los estudios cienciométricos utilizan indicadores relativos para la caracterización temática de la producción científica, para identificar las principales fortalezas y debilidades de un país en un dominio del conocimiento.

Objetivo: hacer la caracterización temática de la producción científica cubana sobre salud indexada en Scopus durante el período 1996-2010, a partir de patrones de especialización temática.

Métodos: estudio descriptivo. Se compilaron datos relativos al número total de documentos indexados por Scopus, así como la productividad y la tasa de crecimiento anual. Se calculó el índice de especialización temática de la producción científica.

Resultados: el índice de especialización temática de la producción científica cubana sobre salud alcanzó niveles superiores con respecto a la región y el mundo. Los mayores índices de especialización se alcanzaron en dominios temáticos donde, a su vez, Cuba exhibe importantes indicadores de salud. Las investigaciones publicadas en revistas nacionales indexadas por Scopus influyeron de manera decisiva en el índice de especialización de las principales categorías temáticas estudiadas.

Conclusiones: las ciencias de la salud de manera general, y particularmente la salud pública, tienen un rol protagónico dentro de la producción científica nacional.

Palabras clave: Cienciometría, indicadores bibliométricos, índice de especialización, Ciencias de la Salud, salud pública, Cuba. 


\section{ABSTRACT}

I ntroduction: scientometric studies use relative indicators for the thematic characterization of scientific production, in order to identify the strengths and weaknesses of a country in a knowledge domain.

Objective: the use of specialization patterns for the thematic characterization of the Cuban scientific production in health indexed by Scopus during the 1996-2010 period. Methods: descriptive study. The total number of documents covered by Scopus as well as the productivity and the annual growth rate were compiled. The thematic specialization index of the scientific production was estimated.

Results: the thematic specialization index of the Cuban scientific production in health achieved higher levels than those of the Latin American region and the world. The highest indexes went to thematic domains where Cuba shows important health indicators. The published research papers in national journals covered by Scopus have a decisive influence on the specialization index of the main studied thematic categories.

Conclusions: health sciences in general, and particularly the public health, have a leading role in the Cuban scientific production.

Key words: Scientometrics, bibliometric indicators, specialization index, health sciences, public health, Cuba.

\section{NTRODUCCI ÓN}

Con el advenimiento del nuevo milenio, el Sistema Nacional de Salud de Cuba se trazó la meta de desarrollar un programa para el fortalecimiento de su sistema de información cientificotécnica, sobre la base de concebir el desarrollo auténtico de la salud pública con un fundamento científico soportado en la capacidad propia para generar soluciones a partir de lo mejor de la práctica y el conocimiento nacional e internacional. $^{1}$

De esta forma, el enfoque hacia la producción de conocimientos, y el reconocimiento del importante rol de los investigadores nacionales en estos procesos, se convirtieron en estrategias de primer orden para una política empeñada en transformar el sistema nacional de información científica en ciencias de la salud en una red descentralizada de servicios y fuentes de información de calidad, sustentada en una red humana altamente calificada y bien informada, con una gran producción científica y comprometida con la salud de la población. ${ }^{1}$

El fortalecimiento de las redes de información, y en especial de los canales de difusión del conocimiento científico generado por los investigadores, fue precisamente uno de los mayores retos de un programa orientado no solo al aumento de los volúmenes de producción científica, sino también al aumento del nivel cualitativo de esta producción científica, así como de su visibilidad internacional.

Tradicionalmente, los estudios nacionales e internacionales realizados con el fin de caracterizar la producción científica nacional en canales de comunicación de máxima visibilidad, habían identificado a las ciencias agrícolas y biológicas como disciplinas predominantes dentro de la actividad científica nacional. ${ }^{2-9}$ De igual forma, el análisis 
de las características y el comportamiento de la producción científica nacional, en comparación con el experimentado en la región latinoamericana, ponía de manifiesto un conjunto de diferencias que hacían considerar a Cuba como un caso atípico en el contexto de la región, por cuanto no era Estados Unidos el principal país colaborador, y no se apreciaba una correspondencia entre el volumen de la producción científica y el potencial de recursos humanos dedicados a actividades científicas y tecnológicas. ${ }^{10}$

Por otra parte, durante aproximadamente 40 años, las bases de datos del Institute for Scientific Information de Filadelfia (hoy Thomson Reuters), fueron prácticamente la única fuente de información para el desarrollo de este tipo de estudios cienciométricos, encaminados a evaluar el comportamiento de los países en los diversos dominios del conocimiento científico. ${ }^{11}$ Sin embargo, el escenario cambió con el surgimiento de Scopus, principal índice de citas de bibliografía científica y académica desarrollado por el gigante editorial Elsevier, en el año 2004. ${ }^{12}$ Su mayor cobertura documental, en comparación con las bases de datos de Thomson Reuters, llamó rápidamente la atención de los tomadores de decisiones en materia de política científica, y entidades internacionales como la Organización para la Cooperación y el Desarrollo Económico (OCDE) comenzó a utilizarla como base para sus análisis de la producción científica mundial. ${ }^{13}$

La utilización de Scopus como fuente de datos cienciométricos ha puesto de manifiesto una perspectiva muy diferente de la producción científica nacional, donde se hace evidente la orientación biomédica de la investigación. ${ }^{7}$ La cobertura de revistas nacionales especializadas en temas de salud por parte de esta base de datos, ha sido sin duda un elemento clave para este comportamiento. Sin embargo, más allá de los datos cuantitativos absolutos, la poca utilización de indicadores relativos para la caracterización temática de la producción científica nacional, es una limitación que se observa en muchas de las investigaciones precedentes, y aún no se han dado a conocer suficientes estudios que caractericen la composición de la producción científica especializada en ciencias de la salud, con vistas a identificar sus principales fortalezas y debilidades.

El presente trabajo tiene como objetivo fundamental, hacer la caracterización temática de la producción científica nacional sobre ciencias de la salud indexada en Scopus, en su contexto mundial y regional; así como la identificación del peso de la investigación sobre salud pública en el volumen total de la producción, a partir del uso de indicadores bibliométricos para determinar patrones de especialización temática durante el período 1996-2010.

\section{MÉTODOS}

Los datos derivados de la base de datos Scopus se obtuvieron a partir de la utilización del portal SCImago J ournal \& Country Rank (SJCR) como fuente de información primaria. EI SJCR constituye una importante herramienta cienciométrica desarrollada por el grupo de investigación español SCImago en el año $2007 .{ }^{14}$ El portal ofrece un conjunto de indicadores cuantitativos de productividad e impacto del volumen total de revistas indexadas en Scopus, así como de todos los países del mundo, no solo de forma general, sino también por áreas y categorías temáticas, a partir de los registros bibliográficos compilados en dicha base de datos.

El análisis de la producción científica mundial, regional y nacional en Scopus en ciencias de la salud, se realizó a partir de la compilación de los datos relativos al número total de documentos indexados por Scopus (Ndoc), así como de la productividad y la tasa de crecimiento anual. Los patrones de especialización fueron 
determinados por medio del índice de actividad utilizado en trabajos previos como índice de esfuerzo o índice de especialización temática. ${ }^{15-17} \mathrm{El}$ índice de actividad o índice de especialización temática (IET) fue originalmente propuesto por Frame en $1977,{ }^{18}$ para caracterizar el esfuerzo relativo de un país en un determinado dominio del conocimiento, a partir de la división del porcentaje mundial de publicaciones del país en el dominio, entre el porcentaje mundial de publicaciones del país en todas las disciplinas.

En este caso, el cálculo del índice de especialización (fórmula) para cada una de las categorías temáticas del área de la medicina se realizó a partir de dos perspectivas diferentes: por un lado, tomando como base la proporción mundial de las categorías; y por otro, tomando como base la proporción con respecto al total mundial de artículos comprendidos en el área de la medicina. En ambos casos, se realizó el cálculo de acuerdo con la siguiente expresión:

$$
\begin{aligned}
& \text { IET }=\frac{\left(N d o c_{\text {país (temática) }} / \operatorname{Ndoc}_{\text {país (total) }}\right)}{\left(N d o c_{\text {mundo (temática) }} / N d o c_{\text {mundo (total) }}\right)} \\
& \text { Ndoc pais/temática: cantidad de documentos de un país indexados } \\
& \text { en un área o categoría temática de Scopus. } \\
& \text { Ndoc pais/total total de documentos de un país indexados en } \\
& \text { Scopus. } \\
& \text { Ndoc mundo/temática: cantidad de documentos indexados en un } \\
& \text { área o categoría temática de Scopus. } \\
& \text { Ndoc mundo/total: total de documentos indexados en Scopus. }
\end{aligned}
$$

Para facilitar la visualización del índice de actividad, se utilizó una escala de valores entre -1 y 1 , donde el 0 representa la posición del mundo en los dominios temáticos estudiados. ${ }^{19}$ Los valores por encima de 0 indicaron una mayor especialización de la producción científica de los países estudiados en el dominio analizado con respecto al mundo.

\section{RESULTADOS}

\section{Patrones de producción y productividad anual}

Las ciencias de la salud son, sin lugar a dudas, el dominio del conocimiento más representativo dentro de Scopus. De los cerca de 24 millones de documentos registrados en esta base de datos en abril de 2012, de acuerdo con la información brindada por el portal SCImago J ournal \& Country Rank, más de 6 millones 400 mil pertenecen a las diferentes categorías temáticas que comprenden el área de la medicina $(27,5 \%)$.

Durante los 15 años comprendidos en el presente estudio, la producción científica mundial en esta área creció de manera sostenida (Fig. 1). El comportamiento de la producción ascendió desde poco más de 340 mil artículos en 1996 hasta cerca de 558 mil artículos en el 2010. No obstante, la proporción anual de trabajos ha descendido desde el $30 \%$ a poco más del $25 \%$, lo cual es producto del gradual crecimiento que han experimentado las 27 áreas temáticas contenidas en Scopus (Fig. 2). 
En la región latinoamericana, si bien la proporción de artículos anual en el área se ha mantenido en niveles muy cercanos al $23 \%$, el crecimiento fue todavía más significativo. De un total de 5215 documentos registrados en 1996, la cifra se multiplicó hasta 21018 trabajos en el 2010, lo cual implica una tasa de crecimiento durante el período del $303 \%$, mayor incluso que la experimentada por el total de la producción científica de la región (280 \%).

En Cuba, este comportamiento, si bien comparte patrones similares de crecimiento, tiene características muy específicas. Más del $40 \%$ de la producción científica nacional durante el período pertenece al área de la medicina $(41,7 \%)$, la cual ha experimentado una tasa de crecimiento del $187 \%$, superior al $140 \%$ experimentado por el total de la producción nacional. Si los 273 artículos en esta área representaban en 1996 el 37,2 \% del total, los 784 artículos publicados en el 2010 constituyeron el $44,6 \%$, lo cual señala hacia un protagonismo cada vez mayor, del dominio biomédico dentro de la actividad científica cubana (Fig. 2).

A diferencia de Latinoamérica, donde la tasa de crecimiento anual nunca ha experimentado valores negativos, en Cuba se han observado irregularidades en el comportamiento de la producción anual (Fig. 2 y Fig. 3). Se observa un período comprendido entre los años 2000 y 2005 , donde no solo se identificaron tasas de crecimiento con valores negativos, sino también proporciones muy por debajo de la media nacional, cercanas al $30 \%$.

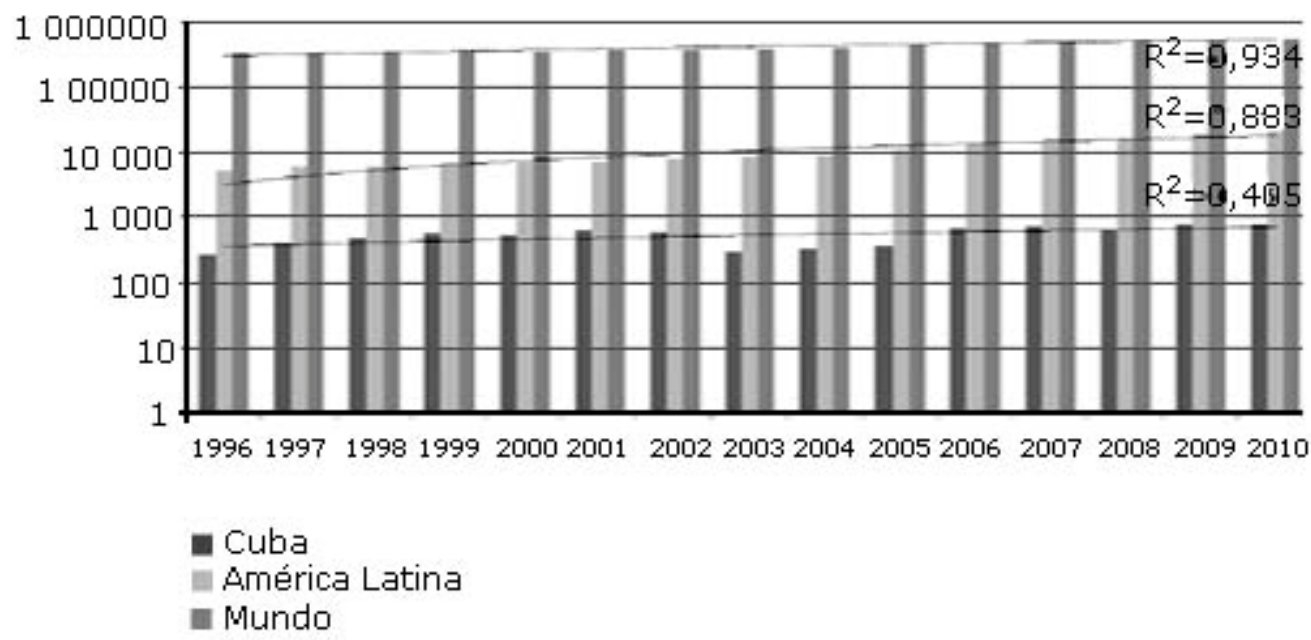

Fig. 1. Crecimiento anual de la producción científica mundial, regional y nacional sobre medicina en revistas visibles internacionalmente (Scopus 1996-2010)

Este decrecimiento de la producción científica, en contraste con el potencial humano existente en Cuba en el área de la salud, no pasó inadvertido para investigadores como Dorta Contreras, quien puso de manifiesto muchos de los problemas que directa o indirectamente afectaban la baja productividad de los profesionales de la salud. ${ }^{20,21}$ En un importante trabajo dado a conocer en el 2006, dicho autor identificó un conjunto de problemas que iban desde la falta de cultura editorial de muchos profesionales, la baja formación en materia de redacción de documentos científicos, las barreras idiomáticas y el excesivo volumen de docencia médica, hasta factores externos como el fallido intento de la administración del entonces presidente norteamericano George W. Bush por detener la publicación de artículos desarrollados por investigadores cubanos en revistas científicas norteamericanas. ${ }^{20}$ 


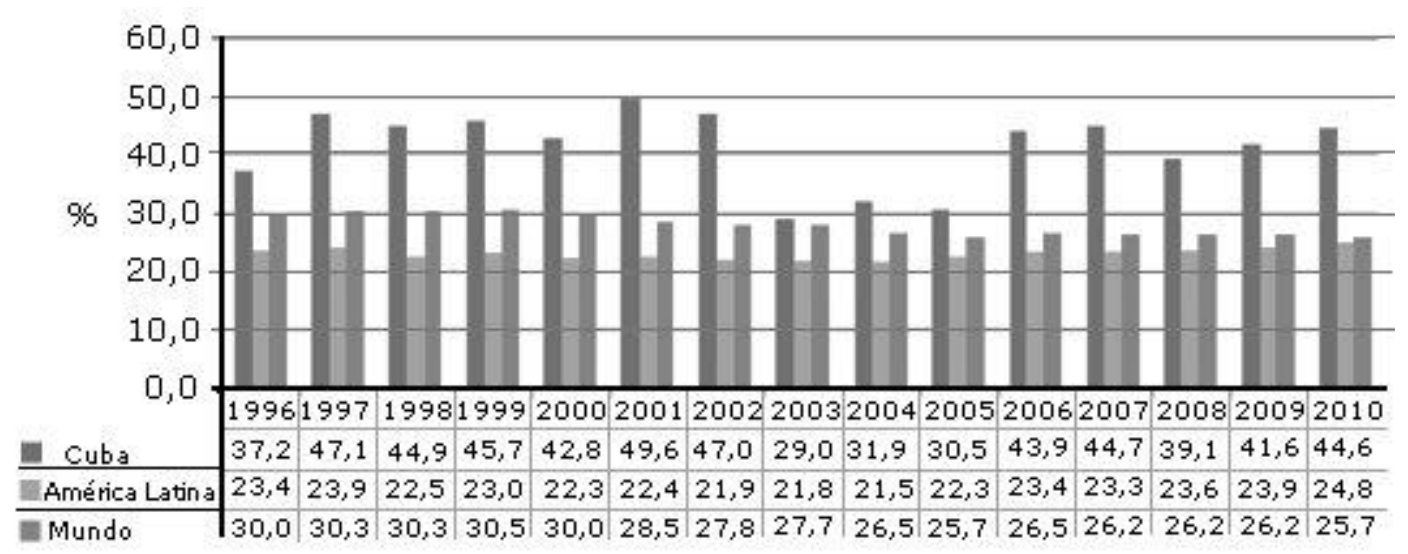

Fig. 2. Proporción con respecto al total de la producción científica mundial, regional y nacional en el área de la medicina (Scopus 1996-2010).

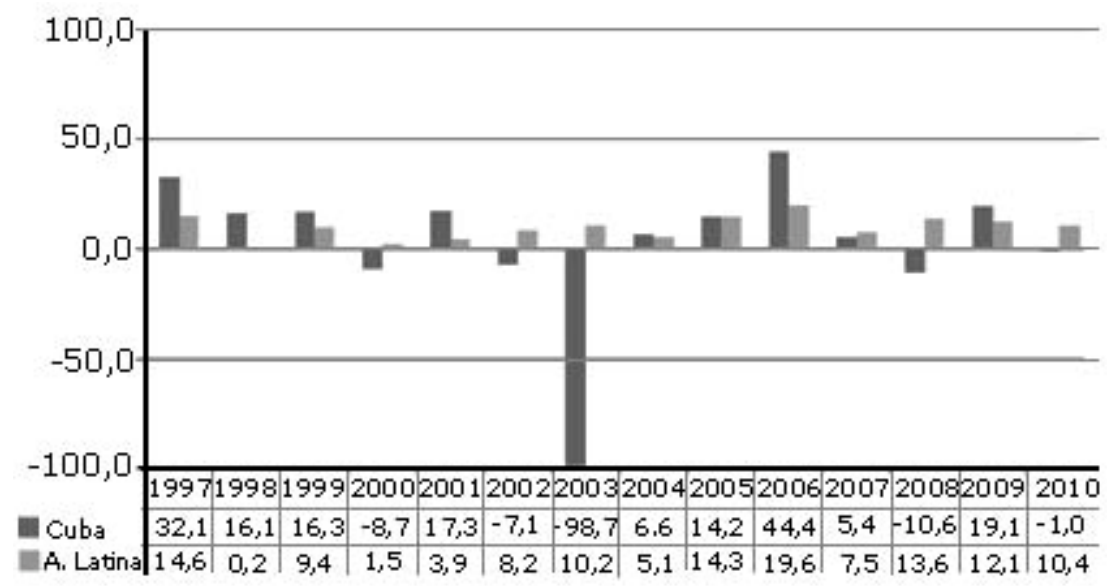

Fig. 3. Tasa de crecimiento anual de la producción científica cubana y latinoamericana sobre medicina (Scopus 1996-2010).

Afortunadamente, el 2006 marcó un punto de retorno a posiciones protagónicas, en el que puede afirmarse que comenzaron a hacerse visibles los efectos del programa para el fortalecimiento del sistema de información cientificotécnica impulsado por el Ministerio de Salud Pública e implementado por el Centro Nacional de Información de Ciencias Médicas y la Red Nacional Infomed. La inclusión en la base de datos Scopus de 19 revistas del área de la salud comprendidas en la colección de revistas científicas SCIELO Cuba, fue sin duda un factor determinante.

\section{Patrones de especialización temática: Cuba en el contexto mundial y regional}

El alcance de los indicadores de producción científica sería insuficiente si solo se limitara a los datos generales de productividad y proporción anual de un determinado nivel de agregación. El ajuste de los indicadores cienciométricos a partir de la utilización de patrones comparativos relativos a otros niveles de agregación permite una aproximación mucho más objetiva a las complejidades que se derivan de las características de la actividad científica. En ese sentido, la utilización del IET ofrece una interesante visión del comportamiento de la producción nacional en ciencias de la salud en relación con el contexto mundial y regional. 
Resulta sumamente clara la alta especialización temática de la producción científica nacional sobre medicina indexada en Scopus. En esencia, el área de la medicina ocupa una posición mucho más protagónica en la producción científica nacional que en la producción científica mundial o regional (Fig.4). La región latinoamericana fue menos especializada que el mundo en temas médicos durante todo el período, aunque en los últimos años de la década se observó la tendencia a comportarse de manera similar. Sin embargo, las verdaderas prioridades de los sistemas nacionales y regionales en relación con el mundo se observan al analizar las diferentes categorías temáticas que componen el área de la medicina (Fig. 5) (Fig.6).

Cuando el IET es calculado con respecto a la producción científica mundial en general, es posible identificar un grupo de categorías que coinciden tanto a nivel regional como nacional (Fig. 5). Ellas son: salud pública, ambiental y ocupacional; obstetricia y ginecología; enfermedades infecciosas; inmunología y alergia; microbiología (médica); y políticas de salud. Por su parte, cuando este es calculado con respecto a la producción científica mundial sobre medicina, a las anteriormente mencionadas se unen las categorías de cirugía, neurología (clínica) y pediatría, perinatología y salud del niño (Fig. 6). Todas estas temáticas engloban un grupo de aspectos que coinciden en las políticas de salud nacional y regional.

De acuerdo con la segunda perspectiva analizada, Cuba se destaca con respecto a la región y al mundo en las categorías medicina (misceláneas), hematología, ortopedia y medicina deportiva, y rehabilitación (Fig. 6). Cuba tiene valores de especialización superiores al mundo y a la región en seis de las diez categorías temáticas con mayor volumen documental cubiertas por Scopus, con excepción de las categorías cardiología y medicina cardiovascular, oncología, radiología y medicina nuclear, y psiquiatría y salud mental, donde se concentra una mayor proporción de la producción científica mundial. Dentro de estas diez categorías, la región alcanza valores de especialización superiores a Cuba solo en el caso de la psiquiatría y salud mental (Fig. 6).

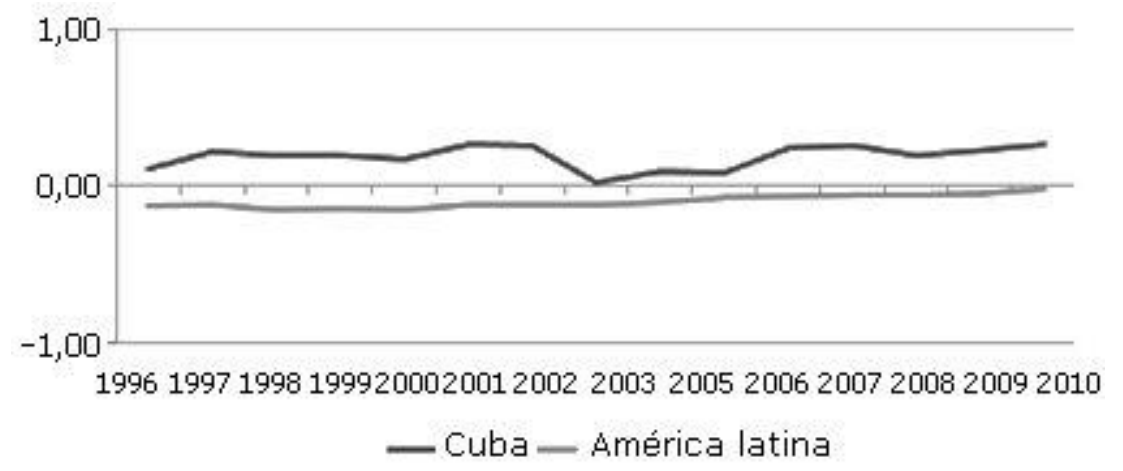

Fig. 4. Especialización temática de la producción científica cubana y latinoamericana en el área de la medicina (Scopus 1996-2010). 
Medicina (miscelaineas? Cardiologia y Medicina Cardiovascular

$$
\text { Cirugia }
$$

Oncologja

Neurologia (climica)

Salud Pública Ambiental y Ocupacional

Psiquialria y Salud Mental

Radiologia y Medicina Huclear

Pediatria, Perinatología y Salud infantil

Obstetricia y Ginecologia

Gastroenterologia

oftalmologia

Patologia y Medicina Forense

Hematologia

Ortopedia y Hedicina Deportiva

Medicina Pulmonar y Respiratoria

Dermatologia

Anestesiologia y Medicina del Dolor

Oterrinolaringologas

Urologia

Farmacoloria imédica

Endocrinologia. Diabetes y Metabolismo

Nefrolosid

Medicina Interna

Enfermedades Infecciosas

Petiabilitación

Genética (clinica

Inmunologia y Alergia

Anatomia

Microbioloziaimedica:

Transplantologia

Politicas de salud

Geriatria v Gerontologia

Cuidados Criticos e Intensivos

Medicina de Emergencia

Reunatologia

Epidemiologia

Medicina Alternativa y Complementaria

Infoumb́lica en Salud

Fisiologia imédica)

Hepatologia

Histologia

Medicina Reproductiva

Bioquimica imedica

Medicina $f$ amiliar

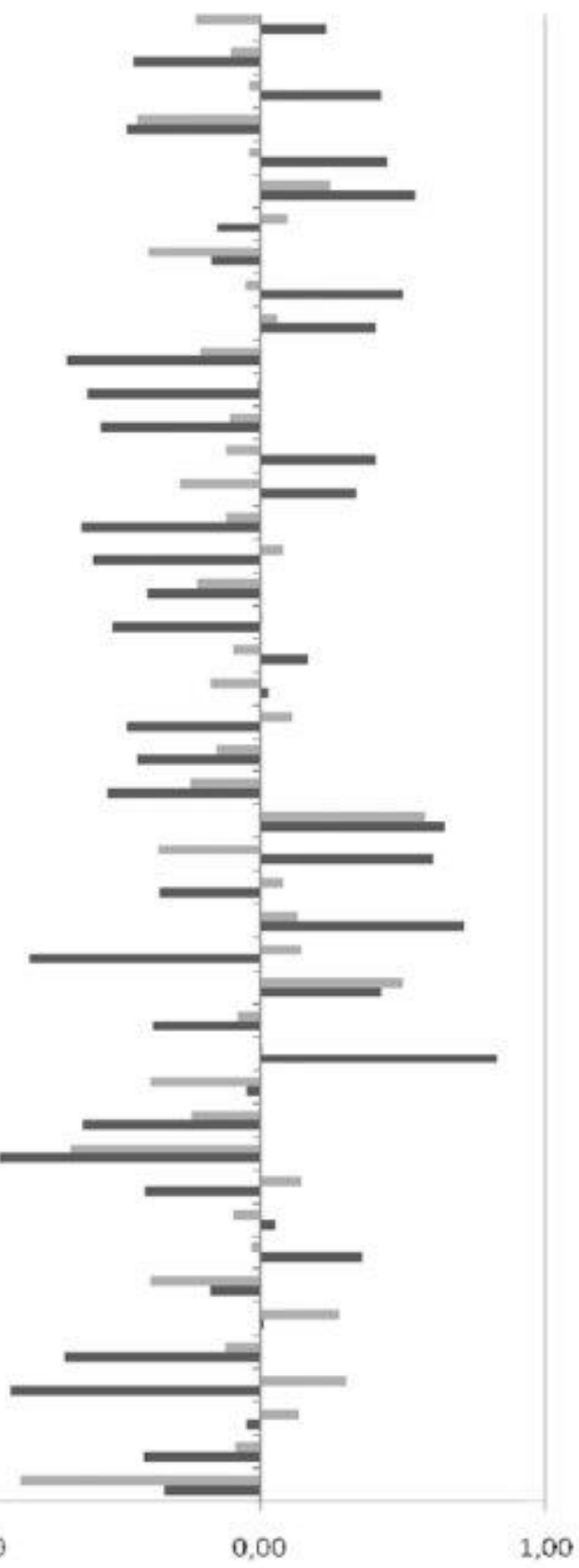

- América latina

Cuba

Fiq. 5. Especialización temática de la producción científica cubana y latinoamericana en las categorias temáticas que componen el área de la medicina con respecto a la producción científica mundial total (Scopus 1996-2010). 


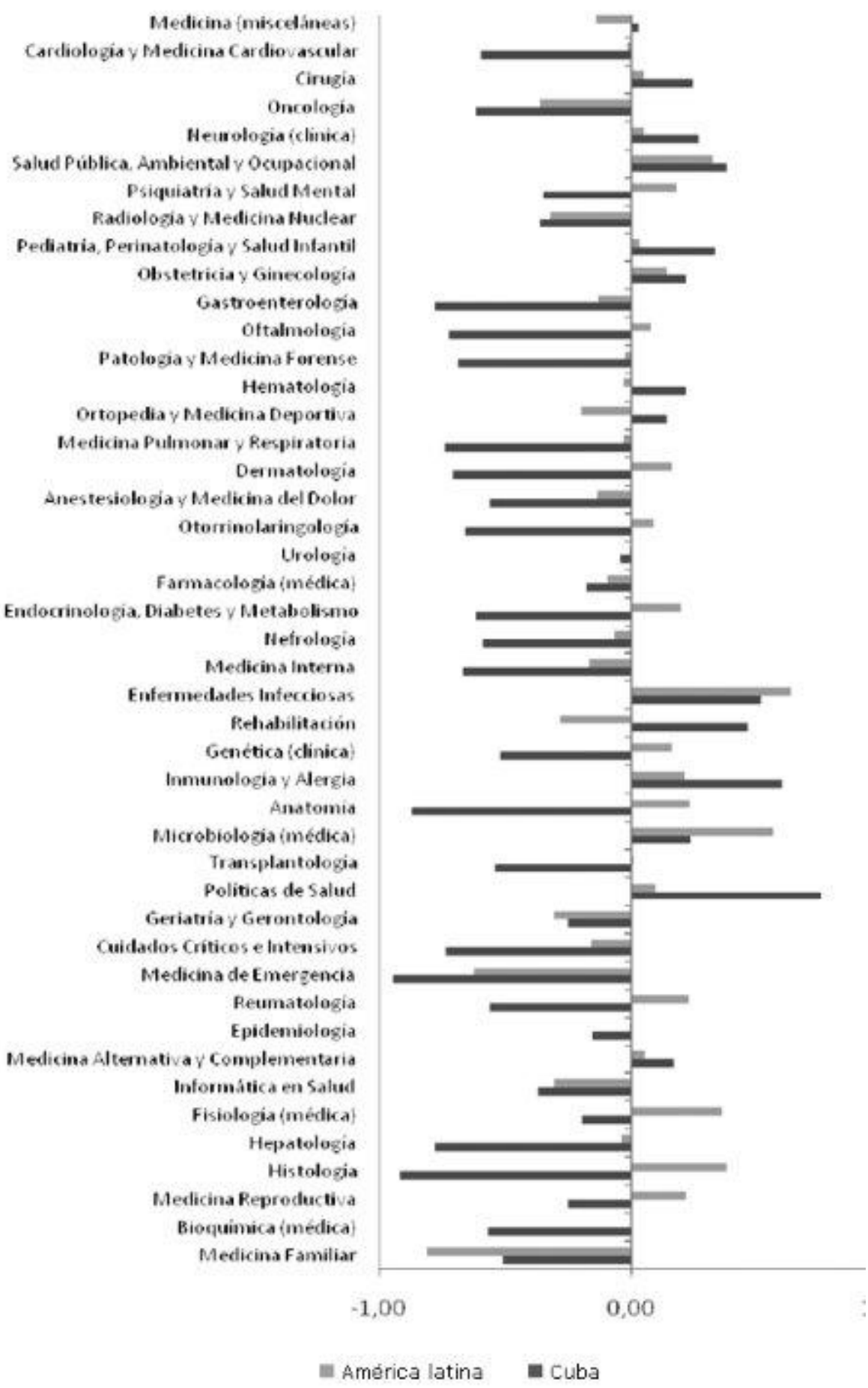

Fig. 6. Especialización temática de la producción científica cubana y latinoamericana en las categorias temáticas que componen el área de la medicina con respecto a la producción científica mundial en el área de la medicina (Scopus 1996-2010).

Los mayores índices de especialización de la producción científica cubana sobre medicina se alcanzaron en las categorías políticas de salud, inmunología y alergia, enfermedades infecciosas, rehabilitación. Sin embargo, dentro de las diez categorías más productivas, la especialización es significativa en el caso de la salud pública, ambiental y ocupacional, la pediatría, perinatología y salud del niño, y la neurología 
(clínica). Todos estos dominios, a nivel nacional, cuentan con sólidos programas nacionales que permiten, a su vez, alcanzar indicadores de salud y de desarrollo humano comparables con los de países ricos e industrializados.

\section{Salud pública como prioridad nacional: evidencia bibliométrica}

El 1,2 \% de la producción científica mundial está indexada bajo la categoría temática salud pública, ambiental y ocupacional, y constituye el 4,4\% del área de la medicina. En América Latina, esta categoría abarca el 2,0\% del total y el 8,6\% del área de la medicina. Sin embargo, en Cuba esta categoría abarca el 4,1 \% del total, y el 9,9\% del área de la medicina, lo cual denota una intensa actividad investigativa.

El análisis de los patrones de especialización de tres categorías temáticas de Scopus estrechamente vinculadas con la salud pública, permite demostrar el rol protagónico del enfoque salubrista dentro del área médica, no solo a nivel nacional, sino también a nivel regional (Fig. 7).
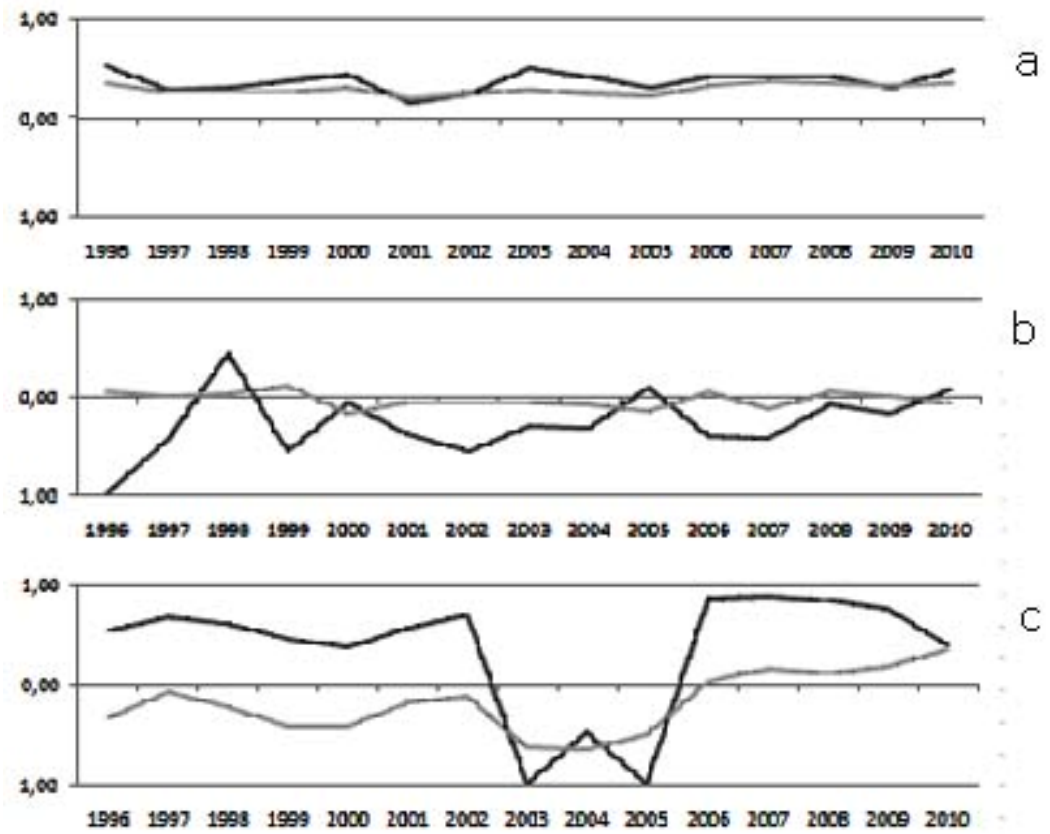

-Cuba-América Latina

Fig. 7. Especialización temática (con respecto al área de la medicina) de la producción científica cubana y latinoamericana en categorías temáticas vinculadas con la salud pública (Scopus 1996-2010). a) Salud Pública, Ambiental y Ocupacional; b) Epidemiología; c) Políticas de Salud.

Tanto Cuba como Latinoamérica tienen un IET superior al mundo en la categoría temática salud pública, ambiental y ocupacional, el cual conserva un comportamiento bastante similar en ambos niveles de agregación durante todo el período comprendido en el presente estudio.

En la categoría epidemiología, la región latinoamericana produce artículos en proporciones muy similares al mundo. De ahí que el índice de especialización relativa conserve valores cercanos a cero durante casi todo el período. A pesar de algunos 
picos de producción significativos en los años 1998, 2000 y 2005, la intensidad de la producción científica cubana en esta categoría está por debajo de la registrada por la región y el mundo, aunque es preciso destacar que tiende gradualmente a ascender durante los últimos 4 años analizados.

Sin embargo, donde se observa un claro dominio de la producción nacional es en la categoría políticas de salud. Con la excepción del período comprendido entre los años 2003 y 2005, la especialización temática de la producción nacional mostró valores muy altos con respecto a la región y el mundo. Es necesario destacar que la región comienza a tener valores de especialización por encima del mundo en esta categoría a partir del 2006, con una clara tendencia al crecimiento sostenido.

A nivel nacional, el hecho de que la Revista Cubana de Higiene y Epidemiología, esté indexada en Scopus solo bajo la categoría salud pública, ambiental y ocupacional, influye decisivamente no solo en los discretos niveles de especialización de la categoría epidemiología, sino también en la alta especialización de la categoría salud pública, ambiental y ocupacional, la cual cuenta, además, con el aporte fundamental de la Revista Cubana de Salud Pública. Por su parte, a la alta especialización de la categoría políticas de salud, contribuye directamente la intensa producción científica durante el período de la Revista Cubana de Información en Ciencias de la Salud (ACIMED). Aunque sus contenidos están estrechamente relacionados con la gestión de la información, ACIMED ha sido un importante órgano de difusión de las principales acciones llevadas a cabo por el Sistema Nacional de Información en Ciencias Médicas.

\section{DISCUSI ÓN}

\section{EL APORTE DECISI VO DE LAS PUBLI CACI ONES SERI ADAS NACIONALES}

La incorporación a Scopus de 19 revistas cubanas editadas por la Editorial de Ciencias Médicas del Ministerio de Salud Pública, órganos de difusión de un gran volumen de producción científica nacional, contribuye a establecer un marcado dominio de las ciencias de la salud sobre el volumen total. Las categorías con mayores índices de especialización, cuentan en su gran mayoría con publicaciones seriadas nacionales cuyos contenidos están cubiertos por Scopus.

Es importante subrayar que la no incorporación de nuevas revistas nacionales pertenecientes a otras áreas temáticas, de conjunto con los niveles actuales de productividad en estas revistas, pudieran provocar un aumento de la proporción de artículos sobre ciencias de la salud, y por ende mayores índices de especialización temática en los próximos años.

Por fortuna, esta presencia sostenida de los contenidos de revistas nacionales en una base de datos de extraordinaria importancia para la comunidad científica mundial, constituye una garantía de visibilidad internacional para la actividad científica nacional dedicada a las ciencias de la salud. Si antes podían sorprender internacionalmente los principales logros del sistema sanitario cubano, hoy pueden corroborarse desde la perspectiva bibliométrica, a partir de la identificación de una alta concentración de investigaciones publicadas que soportan esos resultados, muy especialmente en el ámbito de la salud pública.

Las publicaciones seriadas editadas por el Centro Nacional de Información de Ciencias Médicas, en especial las incluidas en la base de datos Scopus, han comenzado a utilizar el Open Journal Systems (OJS) para la gestión de sus contenidos, y han 
elevado la presencia de expertos internacionales en sus sistemas de arbitraje, con lo cual mejoran los estándares de calidad que influyen en la visibilidad internacional de las revistas. ${ }^{22}$ Sin embargo, aún es necesario comprobar empíricamente que la presencia de estas revistas en repositorios y bases de datos internacionales conlleva un aumento de la citación, lo cual constituirá una interesante línea de investigación futura.

El reto entonces consiste en mantener el crecimiento de los niveles de productividad, elevar el rigor de los procesos de arbitraje, mejorar la calidad de los contenidos publicados, fomentar una cultura de publicación entre médicos y especialistas en ciencias de la salud que pueda derribar cualquier barrera que impida su máxima visibilidad y que estas pautas se extrapolen al resto de revistas cubanas que aún hoy no aparecen en las bases de datos internacionales.

A partir de los resultados obtenidos, puede afirmarse que las ciencias de la salud de manera general, y particularmente la salud pública, tienen un rol protagónico dentro de la producción científica nacional indexada en Scopus durante el período 19962010.

Los patrones de especialización temática de esta producción, y su comportamiento anual en comparación con la región latinoamericana y el mundo, son una clara evidencia de este protagonismo.

Los mayores índices de especialización se alcanzan en dominios temáticos donde, a su vez, se concentran los principales programas nacionales que inciden en el logro de indicadores de salud que prestigian la ciencia cubana a nivel mundial.

El aporte de la investigación publicada en revistas nacionales indexadas por Scopus, es un factor clave para la obtención de altos índices de especialización en las categorías temáticas estudiadas.

\section{Agradecimientos}

Al Grupo de Investigación SCImago. La investigación fue financiada por el Programa de Becas de Corta Duración (IPP-2011-VF-03) del Instituto de Políticas y Bienes Públicos del CSIC, España.

\section{REFERENCI AS BI BLI OGRÁFICAS}

1. Urra González P. Programa para el fortalecimiento del Sistema de Información Científico-Técnica de la Salud en Cuba: una respuesta a los nuevos escenarios. ACIMED [Internet]. 2005 [citado 12 May 2012];13(3). Disponible en: http://scielo.sld.cu/scielo.php? script=sci arttext\&pid=S1024$94352005000300002 \& \operatorname{lng}=\mathrm{es} \& \mathrm{nrm}=\mathrm{iso} \& \mathrm{t} \operatorname{lng}=\mathrm{es}$

2. Sancho R, Bernal G, Galvez L. Approach to the Cuban Scientific Activity by Using Publication Based Quantitative Indicators (19851989). Scientometrics. 1993; 28: 297312. 
3. Licea de Arenas J, Valles J, Williams D. Investigación cubana en agricultura: un enfoque bibliométrico. Ciencias de la Información. 1994; 25: 136141.

4. Torricella Morales RG, Van Hooydonk G, Araujo Ruíz JA. Citation analysis of Cuban research. Part 1. A case study: The Cuban J ournal of Agricultural Science.

Scientometrics. 2000; 47:41326.

5. Guzmán Sánchez MV, Saavedra Fernández O, Sotolongo Aguilar G. Medición de la producción científica en América Latina y el Caribe en el campo agrícola: un estudio bibliométrico. Revista Española Documentación Científica. 2002;25: 15161.

6. Araujo Ruíz JA, Torricella Morales RG, Van Hooydonk G, Arencibia Jorge R. Cuban scientific articles in ISI citation indexes and CubaCiencias databases (19882003). Scientometrics. 2005; 65: 16171.

7. Arencibia Jorge R, Moya Anegón F. Challenges in the study of Cuban scientific output. Scientometrics. 2010;83:723-37.

8. Martí Lahera Y. Ciencia y Género en Cuba (Web of Science, 2001-2007)[tesis]. Granada: Universidad de Granada; 2011.

9. Chinchilla Rodríguez Z. Analysis of Scientific Domains using Scopus-based Scientometric Tools: experiences of the SCImago Research Group. June $28^{\text {th }}$-J uly $1^{\text {st }}$. [Internet]. La Habana: XV International Scientific Congress CNIC, I International Workshop on Scientometric Studies Related to the Biomedical Sciences; 2010 [cited 2012 May 15]. Available from: http://hdl.handle.net/10760/16696

10. Moya Anegón F, Herrero Solana V. Science in America Latina: a comparison of bibliometric and scientifical-technical indicators. Scientometrics. 1999;46:299320.

11. Cameron BD. Trends in the usage of ISI bibliometric data. Portal Libraries Acad. 2005; $5: 10525$.

12. Moya Anegón F, Chinchilla Rodríguez Z, Vargas Quesada B, Corera Álvarez E, González Molina A, Muñoz Fernández GJ, Herrero Solana V. Coverage analysis od Scopus: a journal metric approach. Scientometrics. 2007;73(1):53-78.

13. Tomizawa H. Custom data fuels OECD's innovation strategy. Res Trends. 2008; 8: 3.

14. SCImago Research Group. SCImago J ournal \& Country Rank [Internet]. 2007 [cited 2012 May 15]. Available from: http://www.scimagojr.com/

15. Corera Álvarez E. Análisis del Dominio Científico de las Matemáticas en España: 1990-2004 [tesis]. Granada: Universidad de Granada; 2006.

16. Chinchilla Rodríguez Z. Análisis del Dominio Científico Español: 1995-2002 (ISI, Web of Science) [tesis]. Granada: Universidad de Granada; 2005.

17. Arencibia J orge R. Visibilidad Internacional de la Ciencia y Educación Superior Cubanas: desafíos del estudio de la producción científica [tesis]. Granada: Universidad de Granada; 2010.

18. Frame JD. Mainstream research in Latin America and the Caribbean. Interciencia. 1977; $2: 143-8$.

http://scielo.sld.cu 
19. Glänzel W. Science in Scandinavia: A bibliometric approach. Scientometrics. 2000; 48(2): 121-50.

20. Dorta Contreras AJ . En defensa de nuestra producción científica. ACIMED [Internet]. 2006 [citado 19 Abr 2012]; 14(3): Disponible en: http://scielo.sld.cu/scielo.php?script=sci_arttext\&pid=S102494352006000300015\&lng=es

21. Dorta Contreras AJ. Visibilidad e impacto de las ciencias médicas cubanas: a propósito de los premios anuales de la salud 2006 en artículo científico. ACIMED [Internet]. 2007[citado 19 Abr 2012]; 16(3): Disponible en: http://scielo.sld.cu/scielo.php?script=sci_arttext\&pid=S102494352007000900007\&lng=es

22. Silva Ayçaguer LC. El arbitraje de las revistas médicas, la gestión editorial en red y la calidad de la publicación científica. ACIMED [Internet]. 2011 [citado 15 May 2012];22(2). Disponible en:

http://scielo.sld.cu/scielo. php?script=sci arttext\&pid=S102494352011000200001\&lng=es

Ricardo Arencibia J orge. Centro Nacional de Investigaciones Científicas. Ave. 25 y Calle 158, Cubanacán, Playa 6414. La Habana, Cuba.

Correo electrónico: ricardo.arencibia@cnic.edu.cu 